\title{
Assessment of Microscopic detection of Malaria with Nested Polymerase Chain Reaction in War-torn Federally Administered Tribal Areas of Pakistan
}

\section{Muhammad Faisal Nadeem}

University of Gujrat

Aamer Ali Khattak ( $\square$ amir.khattak@hotmail.com )

University of Haripur https://orcid.org/0000-0002-9400-2984

Adnan Yaqoob

University of Gujrat

Usman Ayub Awan

University of Haripur

Nadia Zeeshan

University of Gujrat

\section{Research}

Keywords: Comparison, diagnostic method, malaria, nested PCR, FATA, Pakistan

Posted Date: January 19th, 2021

DOl: https://doi.org/10.21203/rs.3.rs-148296/v1

License: (c) (i) This work is licensed under a Creative Commons Attribution 4.0 International License.

Read Full License

Version of Record: A version of this preprint was published at Acta Parasitologica on April 11th, 2021. See the published version at https://doi.org/10.1007/s11686-021-00374-8. 


\section{Abstract}

Background: Diagnostic accuracy of malaria is critical for early treatment, control, and elimination of malaria, especially in war-affected malaria endemic areas. Microscopic detection of Plasmodium species has been the gold standard in remote malaria-endemic regions. However, the diagnostic accuracy is still questioned, especially in discriminating mixed and submicroscopic parasitic levels. This study was designed to evaluate the diagnostic performance of microscopic examination against nested PCR analysis in war-torn malaria-endemic Federally Administered Tribal Areas (FATA) of Pakistan.

Methods: Venous blood samples were collected from symptomatic patients for microscopic examination and nested PCR analysis from January 2016 - December 2016 from five Agencies (Bajaur, Mohmand, Khyber, Orakzai and Kurram Agency) and four Frontier Regions (Peshawar, Kohat, Bannu, and Dera Ismail Khan Frontier Region) of FATA. Malaria-positive isolates were confirmed by nested PCR (targeting Plasmodium small subunit ribosomal ribonucleic acid (ssrRNA) genes) for speciation.

Results: Among enrolled participants, 762 were found positive for malaria parasite on microscopic examination of the blood film. P. vivax was found in 623, $P$. falciparum in 132 and 7 were diagnosed with mixed infection ( $P$. vivax and $P$. falciparum coinfection). Nested PCR detected Plasmodium infection in 679 samples (523 P. vivax, 121 P. falciparum, and 35 mixed infections). Compared with microscopy, the sensitivity of nested PCR was $98.94 \%$, and specificity was $98.27 \%$, while the sensitivity and specificity of slide microscopy $89.34 \%$ and $87.99 \%$ respectively.

\section{Conclusion:}

The conventional microscopy method has low sensitivity to detect mixed infection as compared to nested PCR. High sensitivity and specificity observed in nested PCR makes this molecular tool a useful technique for monitoring, controlling, and eliminating malaria-endemic regions.

\section{Background}

The World Health Organization (WHO) malaria report showed an estimated 216 million malaria cases worldwide, with 0.45 million deaths reported in 2017 from 91 countries. It is the second most reported disease in Pakistan, affecting 4.5 million individuals with 33 reported and 1100 estimated deaths in 2016 [1]. More than $80 \%$ of nationwide malaria incidences previously reported were from the war-torn Federally Administered Tribal Area (FATA) [2]. FATA has the highest annual parasite incidence (17.64) in the country (followed by Balochistan and Khyber Pakhtunkhwa provinces) [3], and about 0.1 million cases were screened for malaria in 2016 [4]. Among many factors that influence malaria emergence in this region are political instability, the war against terrorism, mass population displacement within the country (IDPs) and across the border, underprivileged socioeconomic conditions, declining health infrastructure, poor diagnostic, preventive and curative services, regional tribal and sectarian strife all contributing to this huge disease burden [5]. For such remote and under-developed regions, malaria is severe both in local and focal infections [6, 7]. Plasmodium five species (Plasmodium falciparum, Plasmodium vivax, 
Plasmodium ovale, Plasmodium malariae, and Plasmodium knowlesi) are responsible for causing malarial infection in humans. Among the two globally predominant Plasmodium species, $P$. vivax is the most prevalent species in Pakistan that account for $80 \%$ of the malaria cases, while $P$. falciparum causes infection in an estimated $20 \%$ [8]. Incidence of double infections with both $P$. falciparum and $P$. vivax in FATA region has been reported $4-5 \%$ of all malaria infection that is quite high as compared other parts of the country. In FATA region the rate of co-infection with $P$. falciparum and $P$. vivax is quite higher ( $4 \%$ to $6 \%$ ) than other malaria endemic regions of the Pakistan [5]. Diagnosis and surveillance of malaria in a region where more than one species of Plasmodium genus is present are difficult to achieve without using a more sensitive, specific, and reliable diagnostic technique.

Even though empirical diagnosis results in overtreatment due to inaccuracy, it remains the basis for malaria diagnosis in a majority of the symptomatic patients in malaria-endemic regions, where advanced diagnostic facilities are lacking. Nevertheless, a clinical or presumptive diagnosis is the least expensive and most commonly used method that forms the basis for self-treatment in areas such as FATA [9].

A variety of diagnostic tools are available for Plasmodium identification and speciation, including microscopy (light and fluorescence), immuno-chromatographic techniques, nucleic acid amplification methods (PCR and isothermal amplification), and flow cytometry [10]. Dielectrophoretic and magnetophoretic approaches are also among the promising new techniques, but their sensitivity and specificity still has to be evaluated [11]. Laboratory diagnosis of malaria at the primary healthcare level in remote areas is limited to light microscopy. However, this technique has some advantages and shortcomings in proper malaria diagnosis [12]. To overcome the shortcomings of microscopy, a rapid diagnostic test (RDT) has been developed for malaria detection, which is claimed to be simple, accurate, and cost-effective diagnostic tests for malaria. Like microscopy, RDT also has limitations [13], particularly in differences in sensitivity among various kits [14].

During the last decade, the molecular diagnostic technique Polymerase Chain Reaction (PCR) (amplification of $18 \mathrm{~S}$ rRNA genes) has gained popularity over conventional microscopic technique and RDT [15]. The PCR technique has demonstrated its high specificity and sensitivity in Plasmodium identification and speciation [15-18]. Besides detection and mixed-species identification [19-21], PCR has also proven its remarkable utility in follow-up studies on anti-malarial efficacy, molecular epidemiology, and drug resistance studies [7, 8, 13,22]. PCR has been proven to be superior, but the molecular diagnostic setup is not always possible as it is expensive, time-consuming, needs expertise, supervision, and resources [23]. That's why PCR-based malaria diagnosis may not be feasible in remote rural areas like FATA [24].

As per WHO malaria guidelines, all suspected cases must undergo laboratory diagnosis using microscopy and RDT before any therapeutic care because information regarding the effectiveness of presumptive diagnosis is inadequate. This study was conducted to evaluate the performance of nested PCR in the detection of malarial parasites in positive microscopy samples in a warn-torn, remote malariaendemic region of Pakistan. 


\section{Methods}

The study population was comprised of patients visiting healthcare facilities in five Agencies (Bajaur, Mohmand, Khyber, Orakzi, and Kurram Agencies) and four Frontier Regions (Peshawar, Kohat, Bannu, and Dera Ismail Khan Frontier Region) of FATA, Khyber Pakhtunkhwa province from January 2016 to December 2016, as shown in Fig. 1. Ethical clearance for this study was obtained from the Institutional Review Board of the University of Gujrat, Pakistan. Inclusion criteria was malaria febrile patients irrespective of age and gender (having fever, chills, nausea, vomiting, sweats, headache, and fatigue) willing to give oral or written consent (parents or guardians for those below 18 years) to be part of the research. Individuals who were already taking anti-malarial drugs in the past two weeks or those who did not consent to be part of the study were excluded.

$3 \mathrm{~mL}$ of venous blood was collected from patients in vacutainer tube containing EDTA.Thick and thin blood films were prepared, stained with $3 \%$ Giemsa staining solution and observed under the $100 x$ objective of the microscope at study site $[25,26]$. 50uL of whole blood from microscopy positive patients was spotted on properly labeled 3MM Whatman filter paper, air-dried separately to avoid contamination, and were then placed in a zip lock bag with a desiccant for future analysis

For molecular analysis, the Plasmodium genomic DNA was extracted from dried blood spots on the Whatman filter paper by using a previously published method [27]. PCR ingredients and thermal cycler conditions were adapted from previously published research work [8]. MRA-178, 3D7/HB3/Dd2, MRA-179, and MRA-180 were used as positive controls for $P$. vivax, $P$. falciparum, $P$. malariae, and $P$. ovale, respectively, while for the negative control, ultra-pure water was used. The PCR products were resolved by electrophoresis, stained with ethidium bromide, and visualized with ultraviolet illumination.

DAG_Stat online statistics software was used to evaluate diagnostic tests like sensitivity, specificity, predicted values, and $95 \%$ confidence intervals $(\mathrm{Cl})$. Vassar Stats tool for performing statistical computation was used to estimate Kappa coefficients (http://vassarstats.net/) [28]. The kappa values were used to classify the strength of agreement between each microscopy and nested PCR. Additionally, Standard formulae were used to calculate positive predictive values [TP/(TP + FP)] negative predicted values $[T N /(F N+T N)]$ and test accuracy $[(T P+T N) /$ number of all tests]. For diagnostic accuracy to avoid the risk of bias due to shortcomings in design and conduct, STARD 2015 guidelines of Reporting of Diagnostic Accuracy were followed whenever required during analysis [29].

\section{Results}

Among the malaria suspected population, 762 people were found positive on microscopic examination of Giemsa-stained blood films. P. vivax parasite was found in 623(82\%), 132(17\%) were positive for $P$. falciparum, and $7(1 \%)$ had mixed infection (a mixed infection counting for both $P$. vivax and $P$. falciparum species as positive), as illustrated in Fig. 3.

All microscopy tested Plasmodium positive samples were subjected to parasitic DNA extraction to identify Plasmodium species using PCR. In the first round of nested PCR the product size was $1100 \mathrm{bp}$. 
Agarose gel electrophoresis revealed a 205 bp amplification product for P. falciparum, 120 bp for P. vivax, 144 bp for P. malariae and 800 bp DNA P. ovale in parallel with 100 bp DNA ladder as shown in Fig. 2.

However, discordant results were observed among several malaria positive isolates, and discrepancies of Plasmodium species were observed between results of both techniques, which are depicted in Table 1. A total of $523(77 \%)$ microscopy positive malarial isolates were identified as $P$. vivax by nested PCR, $121(18 \%)$ were confirmed as $P$. falciparum, 35(5\%) were found to harbor mixed infection and 83 samples tested negative, as shown in Fig. 2. None of the samples tested positive for $P$. malariae or $P$. ovale, Slides were not available for re-examination to resolve discrepancies, but DNA was re-extracted, and nested PCR was repeated.

Table 1

Cross-tabulation between microscopy and nested polymerase chain reaction for Plasmodium parasite detection and speciation in Federally Administered Tribal Area, Pakistan.

\begin{tabular}{|ll|}
\hline \multicolumn{2}{|l|}{ Plasmodium parasites detection by microscopy and nested-PCR $\left(\mathbf{n}^{\mathbf{t}}=762\right)$} \\
\hline Microscopy Results & Nested-PCR Results \\
\hline P. vivax (623) & P. vivax (518), P. falciparum (19), Mixed (15), Negative (71) \\
\hline P. falciparum (132) & P. vivax (5), P. falciparum (98), Mixed (18), Negative (11) \\
\hline Mixed (7) & P. vivax (0), P. falciparum (4), Mixed (2), Negative (1) \\
\hline${ }^{\mathrm{t}} \mathbf{n}=$ number of positive blood samples examined. \\
\hline
\end{tabular}

To compare the agreement between microscopy and PCR, Cohen's kappa coefficient was used, which showed a high (kappa $=0.89)$ agreement for the presence or absence of Plasmodium infection. Nevertheless, for Plasmodium species, the coinfection agreement was low (kappa $=0.51$ ) because PCR identified 33(7\%) samples as mixed-species infections, which were declared mono-species infections by microscopy. The agreement between the diagnostic tools, microscopy, and PCR in diagnosis as $P$. falciparum and $P$. vivax were kappa $=0.84$.

PCR Sensitivity and Specificity were calculated by using microscopy as a reference method. Sensitivity of PCR was $98.94 \%(95 \% \mathrm{Cl}=97.93-99.54)$ and specificity was $98.27 \%(95 \% \mathrm{Cl}=96.99-99.10)$.

Comparatively, microscopic analysis seemed to be less specific with specificity $87.99 \%(95 \% \mathrm{Cl}=85.33-$ 90.32) and sensitivity $89.34 \%,(95 \% \mathrm{Cl}=86.93-91.45)$, as shown in Table 2. Test of the agreement was calculated by comparison of PCR with blood film examination. PCR showed greater specificity and sensitivity over the manual microscopic examination. 
Table 2

Comparative analysis of both microscopy and nested-PCR for the diagnosis of malaria.

\begin{tabular}{|lllll|}
\hline & \multicolumn{2}{l}{ Microscopy } & \multicolumn{2}{l|}{ Nested-PCR } \\
\hline & Value & $95 \% \mathrm{Cl}$ & Value & $95 \% \mathrm{Cl}$ \\
\hline Sensitivity & 89.34 & $86.93-91.45$ & 98.94 & $97.93-99.54$ \\
\hline Specificity & 87.99 & $85.33-90.32$ & 98.27 & $96.99-99.10$ \\
\hline Positive Likelihood Ratio & 7.44 & $6.07-9.11$ & 57.14 & $32.61-100.13$ \\
\hline Negative Likelihood Ratio & 0.12 & $0.10-0.15$ & 0.01 & $0.01-0.02$ \\
\hline Positive Predictive Value & 89.11 & $86.97-90.93$ & 98.43 & $97.27-99.10$ \\
\hline Negative Predictive Value & 88.24 & $85.91-90.23$ & 98.84 & $97.71-99.41$ \\
\hline Accuracy & 88.7 & $86.95-90.28$ & 98.62 & $97.88-99.16$ \\
\hline
\end{tabular}

\section{Discussion}

Malaria is the second most reported disease in Pakistan, with about $98 \%$ of the population at varying risk of contracting malaria infection and about $29 \%$ living in highly endemic areas, including FATA [4]. These Plasmodium endemic regions of Pakistan share borders with WHO-declared malaria-endemic countries Iran and Afghanistan [5]. Malaria surveillance data has been made available to national and provincial malaria control programs by a web of diagnostic centers established by local government and international donor agencies[4]. FATA is the most neglected part of Pakistan in terms of healthcare facilities. Health Directorate is chiefly responsible for providing basic health services, but the healthcare systems have been badly affected due to constant military conflicts, political unrest in the neighborhood Afghanistan and the ongoing war against terror. [6]. Malaria is the leading cause of morbidity in the FATA region, which contributes $80 \%$ of the disease burden of malaria in Pakistan with the highest test positivity rate (17.01\%) and API (17.64) [30]. Many epidemiological studies have reported a high prevalence of $P$. vivax infection, 80-90\% compared to $P$. falciparum 10-20\% [3]. Incidence of double infections with both $P$. falciparum and $P$. vivax in FATA region has been reported $4-5 \%$ of all malaria infection that is quite high as compared other parts of the country [5]. However, 363 malaria diagnostic centers established in FATA for a population of about 5 million can't meet the public health demand for malaria management. A presumptive diagnosis seems the only solution in such an area for treating malaria patients [31].

Although Giemsa-stained blood film microscopy has been recommended as a gold standard malaria diagnostic technique, in those areas where microscopy is not possible (due to unavailability of either microscope or trained staff), prompt malaria confirmation can be done using rapid diagnostic tests (RDTs). Microscopic examination has advantages such as low direct costs, sensitivity to differentiate between malaria species, and the ability to measure parasitemia level before and after treatment. 
However, many factors affect the quality of results generated through microscopic examination of blood films. These include parasitemia detection level per microliter of blood, species identification constraints, insufficient staff, competency level of staff, substandard or inappropriate equipment. These factors contribute towards reporting of false-negative and false-positive results and discrepancies in species identification using microscopy. In the present study, microscopic examination resulted in a misdiagnosis of several mixed specie infections. The differences in sensitivity and specificity of microscopy and PCR are obvious through differences in their reported positivity rate in literature [19][1]. A study reported significant microscopy shortcomings with substantial problems even in reasonably sophisticated laboratories. Studies concluded that strict quality assurance protocols must be enforced to maintain microscopic examination accuracy $[26,27]$.

In this study, some discrepancies were found in microscopy results after confirmation with nested PCR. Additionally, PCR has been considered a molecular tool for Plasmodium identification and species discrimination. This technique helps in the detection of low parasitemia levels in samples, speciation and significantly robust in identifying mixed species infection that is mostly missed by routine microscopy. Nested PCR revealed that $83(11 \%)$ of microscopy positive malaria cases were negative which is probably due to low quality of stained smears, presence of artefacts, wrong diagnosis, slide contamination [32].

In cases of mixed infection diagnosis, 7 (1\%) samples were mixed by microscopy whereas nested PCR detected $35(5 \%)$ samples harboring mixed infections. Zaman et al. reported that a major drawback in blood film examination is the misdiagnosis of Plasmodium species, mainly in case of mixed infection [20]. A similar finding is observed in the study conducted in India where microscopy declared 6 mixed species infection, but isolated when exposed to PCR revealed 12 [10]. Detection of malarial DNA by PCR technology has much greater sensitivity and specificity, particularly in mixed infections and in case of low parasitemia [21-23].

The present study has reported high sensitivity and specificity of PCR $[24,25]$. In contrast, a study reported the less sensitivity and specificity of PCR lower than the current study [26]. PCR also has few disadvantages like it is time-consuming, involves many steps, requires technical staff, expensive because of the complex equipment needed to run the PCR which hinder PCR implementation as an alternative to microscopy in remote areas and low-income countries.

The results of microscopy compared with nested PCR revealed that PCR has higher sensitivity and specificity for detection of malarial parasites. All performance characteristics like sensitivity, specificity, positive predictive value, negative predictive value and accuracy were higher for nested PCR than microscopy. We recommend using PCR for the detection of Plasmodium infection and also for specie differentiation. PCR-based methods are useful in detecting malaria parasite, but recently this molecular tool also showed its supremacy in evaluating treatment monitoring response, molecular drug resistance, and parasite insecticide resistance detection.

\section{Conclusion}


Our study reported that PCR detected Plasmodium and discriminated very well between different species when compared to microscopy. Nested PCR is very effective in detecting mixed species infection than microscopy. There is a need for PCR detection as missed or misread infections by microscopists are very common in areas where more than one Plasmodium species co-exist. Higher sensitivity and specificity associated with the nested PCR, molecular detection method holds enormous promise for malaria diagnosis and can be used as effective monitoring tools for surveillance, control, and malaria elimination from a remote area like FATA. Based on the findings, we strongly recommend using the PCR technique as the reference method in government institute and diagnostic center to diagnose malarial parasite and verify blood film results and investigate the drug-resistant strains of plasmodium species.

\section{Declarations}

Acknowledgment: The authors are thankful to Dr. Aamer Ali Khattak, Dr. Huma Fatima, Mr. Adnan Yaqoob, and other Lab staff to prepare, keep, and store samples properly. Special thanks to the Genomic Lab staff for cooperating in extraction, amplification of DNA, and DNA analysis.

Author Contributions: MFN and AAK conceived, designed the paper, and supervised the research. AAK, AY, and UAA wrote the manuscript. All the authors read and approved the manuscript.

\section{Funding:}

None to declare

\section{Availability of Data Material:}

All the relevant data is available and can be provided upon request.

\section{Ethical Approval:}

Ethical approval is obtained from the ethical committee, Department of Medical Laboratory Technology, University of Haripur, Haripur, Khyber Pakhtunkhwa, Pakistan

\section{Conflict of Interest:}

The authors have no conflict of interest to declare.

\section{References}

1. Organization WH. World Malaria Report-2017. WHO Global Malaria Programme. 2018.

2. Hussain K, Shafee M, Khan N, Jan S, Tareen A, Khan M. Seroprevalence of Pediatric Malaria in Quetta, Balochistan, Pakistan. In Iran J Parasitol. Volume 82013: 342-347.

3. Organization WH: Global Technical Strategy for Malaria 2016-2030. Geneva: World Health Organization; 2015. 2017. 
4. Control DoM: Malaria Annual Report 2016. pp. 49. Pakistan: Program Management Unit Directorate of Malaria Control; 2016:49.

5. Karim AM, Hussain I, Malik SK, Lee JH, Cho IH, Kim YB, et al. Epidemiology and clinical burden of malaria in the war-torn area, Orakzai Agency in Pakistan. PLoS Negl Trop Dis. 2016;10:e0004399.

6. Khan AQ, Ali I, Imran M, Yaseen M, Abbas SZ, Mufti FUD, et al. Epidemiological study in FATA areas of Khyber-Pakhtunkhwa, Pakistan. The Professional Medical Journal. 2016;23:553-8.

7. Khattak AA, Venkatesan M, Jacob CG, Artimovich EM, Nadeem MF, Nighat F, et al. A comprehensive survey of polymorphisms conferring anti-malarial resistance in Plasmodium falciparum across Pakistan. Malar J. 2013;12:300.

8. Khattak AA, Venkatesan M, Nadeem MF, Satti HS, Yaqoob A, Strauss K, et al. Prevalence and distribution of human Plasmodium infection in Pakistan. Malar J. 2013;12:297.

9. Ojurongbe O, Adegbosin OO, Taiwo SS, Alli OAT, Olowe OA, Ojurongbe TA, et al: Assessment of clinical diagnosis, microscopy, rapid diagnostic tests, and polymerase chain reaction in the diagnosis of Plasmodium falciparum in Nigeria. Malaria research and treatment. 2013;2013.

10. Siwal N, Singh US, Dash M, Kar S, Rani S, Rawal C, et al. Malaria diagnosis by PCR revealed differential distribution of mono and mixed species infections by Plasmodium falciparum and $P$. vivax in India. PLoS One. 2018;13:e0193046.

11. Kasetsirikul S, Buranapong J, Srituravanich W, Kaewthamasorn M, Pimpin A. The development of malaria diagnostic techniques: a review of the approaches with focus on dielectrophoretic and magnetophoretic methods. Malar J. 2016;15:358.

12. Santana-Morales MA, Afonso-Lehmann RN, Quispe MA, Reyes F, Berzosa P, Benito A, et al. Microscopy and molecular biology for the diagnosis and evaluation of malaria in a hospital in a rural area of Ethiopia. Malar J. 2012;11:199.

13. Wongsrichanalai C, Barcus MJ, Muth S, Sutamihardja A, Wernsdorfer WH. A review of malaria diagnostic tools: microscopy and rapid diagnostic test (RDT). Am J Trop Med Hyg. 2007;77:119-27.

14. Performance MRDT. Results of WHO product testing of malaria RDTs: Round 1. Geneva: World Health Organization; 2008.

15. Pinheirob VE, Thaithongc S, Browna KN. High sensitivity of detection of human malaria parasites by the use of nested polymerase chain reaction. Mol Biochem Parasitol. 1993;61:315-20.

16. Kain KC, Harrington MA, Tennyson S, Keystone JS. Imported malaria: prospective analysis of problems in diagnosis and management. Clin Infect Dis. 1998;27:142-9.

17. Singh B, Bobogare A, Cox-Singh J, Snounou G, Abdullah MS, Rahman HA. A genus-and speciesspecific nested polymerase chain reaction malaria detection assay for epidemiologic studies. Am J Trop Med Hyg. 1999;60:687-92.

18. Snounou G, Pinheiro L, Gonçalves A, Fonseca L, Dias F, Brown KN, et al. The importance of sensitive detection of malaria parasites in the human and insect hosts in epidemiological studies, as shown by the analysis of field samples from Guinea Bissau. Trans R Soc Trop Med Hyg. 1993;87:649-53. 
19. Krishna S, Bharti PK, Chandel HS, Ahmad A, Kumar R, Singh PP, et al. Detection of mixed infections with Plasmodium spp. by PCR, India, 2014. Emerging Infect Dis. 2015;21:1853.

20. Makler MT, Palmer CJ, Ager AL. A review of practical techniques for the diagnosis of malaria. Ann Trop Med Parasitol. 1998;92:419-34.

21. Zakeri S, Kakar Q, Ghasemi F, Raeisi A, Butt W, Safi N, et al. Detection of mixed Plasmodium falciparum \& P. vivax infections by nested-PCR in Pakistan, Iran \& Afghanistan. Indian J Med Res. 2010;132:31-5.

22. Khattak AA, Venkatesan M, Khatoon L, Ouattara A, Kenefic LJ, Nadeem MF, et al. Prevalence and patterns of antifolate and chloroquine drug resistance markers in Plasmodium vivax across Pakistan. Malar J. 2013;12:310.

23. Tangpukdee N, Duangdee C, Wilairatana P, Krudsood S. Malaria diagnosis: a brief review. Korean J Parasitol. 2009;47:93.

24. Hänscheid T, Grobusch MP. How useful is PCR in the diagnosis of malaria? Trends Parasitol. 2002;18:395-8.

25. Organization WH: Malaria microscopy quality assurance manual-version 2. World Health Organization; 2016.

26. Organization WH: Basic Malaria Microscopy: Tutor's guide. World Health Organization; 2010.

27. Zainabadi K, Adams M, Han ZY, Lwin HW, Han KT, Ouattara A, et al. A novel method for extracting nucleic acids from dried blood spots for ultrasensitive detection of low-density Plasmodium falciparum and Plasmodium vivax infections. Malar J. 2017;16:377.

28. Mackinnon A. A spreadsheet for the calculation of comprehensive statistics for the assessment of diagnostic tests and inter-rater agreement. Comput Biol Med. 2000;30:127-34.

29. Bossuyt PM, Reitsma JB, Bruns DE, Gatsonis CA, Glasziou PP, Irwig LM, et al. Towards complete and accurate reporting of studies of diagnostic accuracy: the STARD initiative. Clin Chem Lab Med. 2003;41:68-73.

30. Control DoM. Pakistan Malaria Annual Report 2019. Pakistan: Program Management Unit Directorate of Malaria Control; 2019.

31. Okoro C, Chukwuocha U, Nwakwuo G, Ukaga C: Presumptive diagnosis and treatment of malaria in febrile children in parts of south eastern Nigeria. Journal of Infectious Diseases \& Therapy. 2015.

32. Shahwani Z, Aleem A, Ahmed N, Mushtaq M, Afridi S. A PCR method based on 18S rRNA gene for detection of malaria parasite in Balochistan. JPMA. 2016;66.

\section{Figures}




\section{Federally Administered Tribal Areas (FATA), Pakistan}

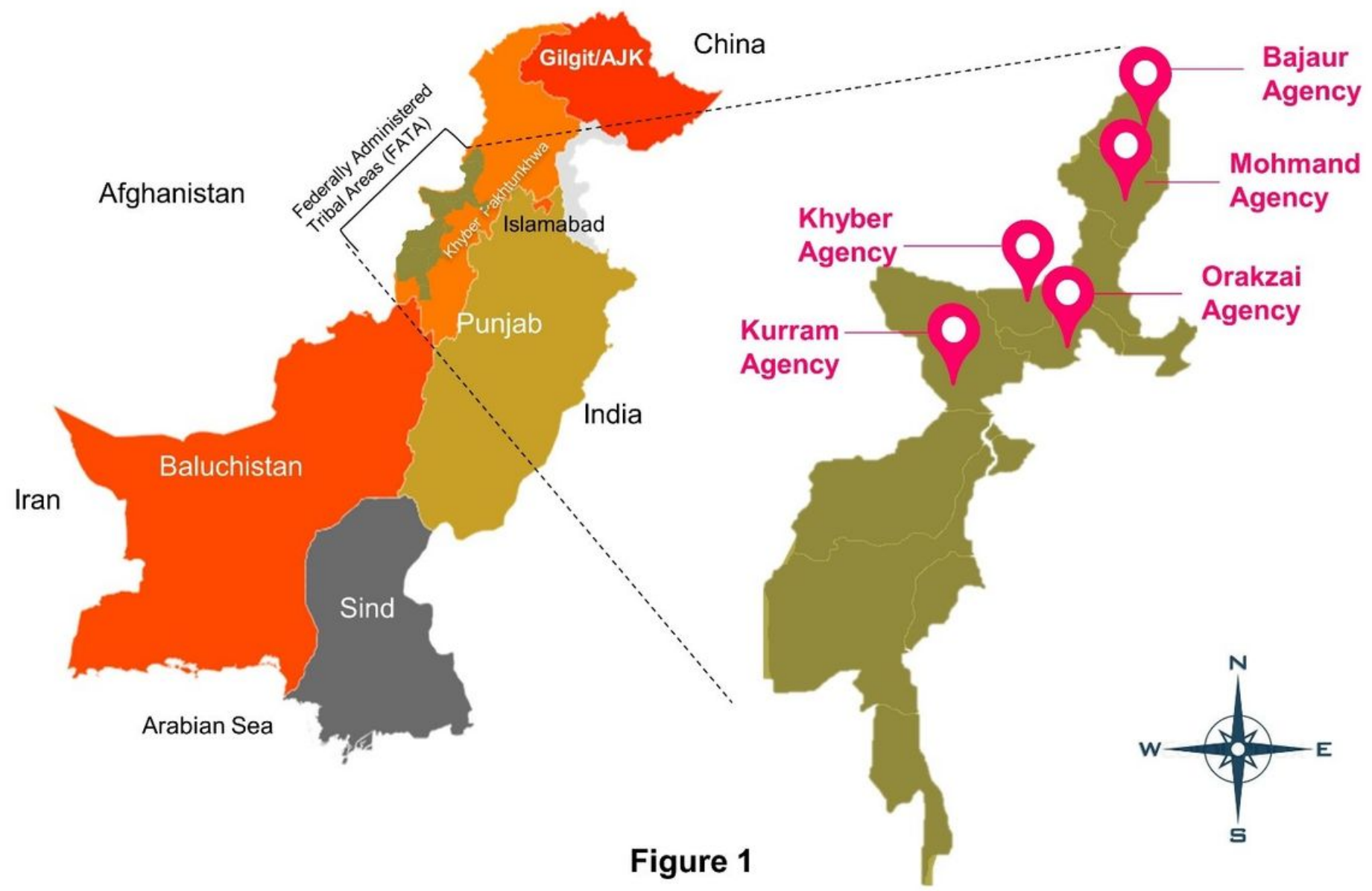

\section{Figure 1}

Sample sites from different agencies in war-torn Federally administered tribal areas (FATA) of Pakistan Note: The designations employed and the presentation of the material on this map do not imply the expression of any opinion whatsoever on the part of Research Square concerning the legal status of any country, territory, city or area or of its authorities, or concerning the delimitation of its frontiers or boundaries. This map has been provided by the authors. 


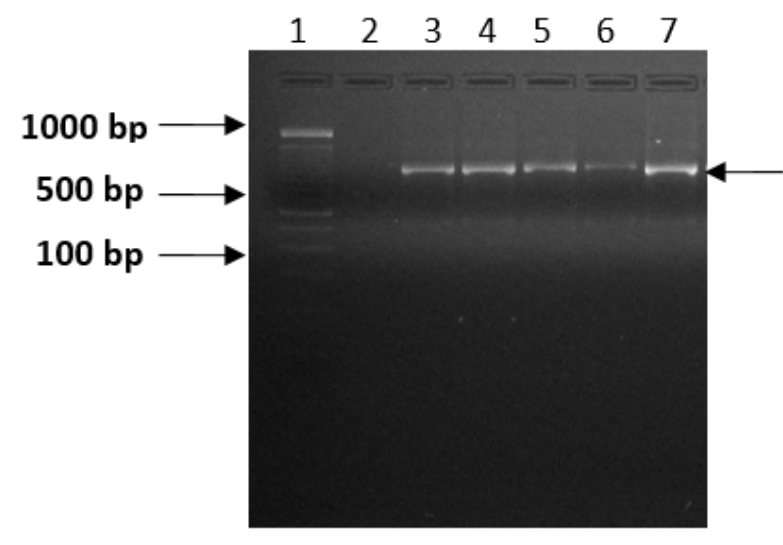

(a) Plasmodium (Genus)

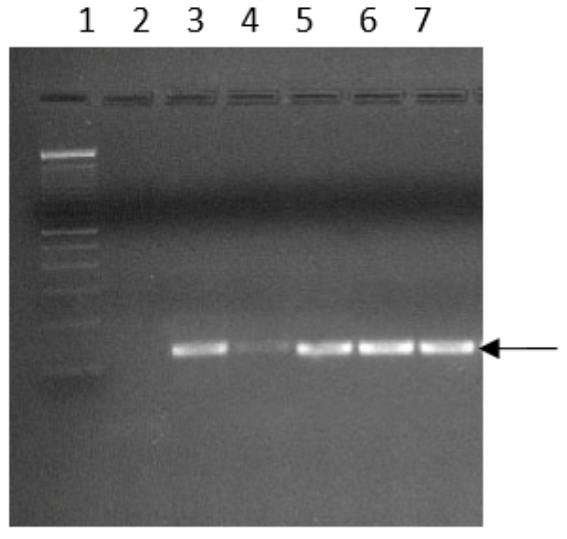

(b) Plasmodium vivax

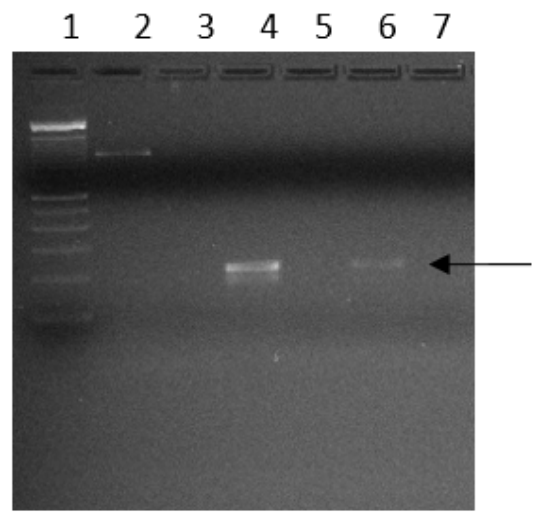

(c) Plasmodium falciparum

Note: None of the isolate showed $P$. malariae and $P$. ovale species

\section{Figure 2}

Nested PCR results (a) genus Plasmodium (b) Plasmodium vivax and (c) Plasmodium falciparum Lane 1: 100 bp ladder, Lane +C: Positive control, Lane $-\mathrm{C}$ : Negative control.

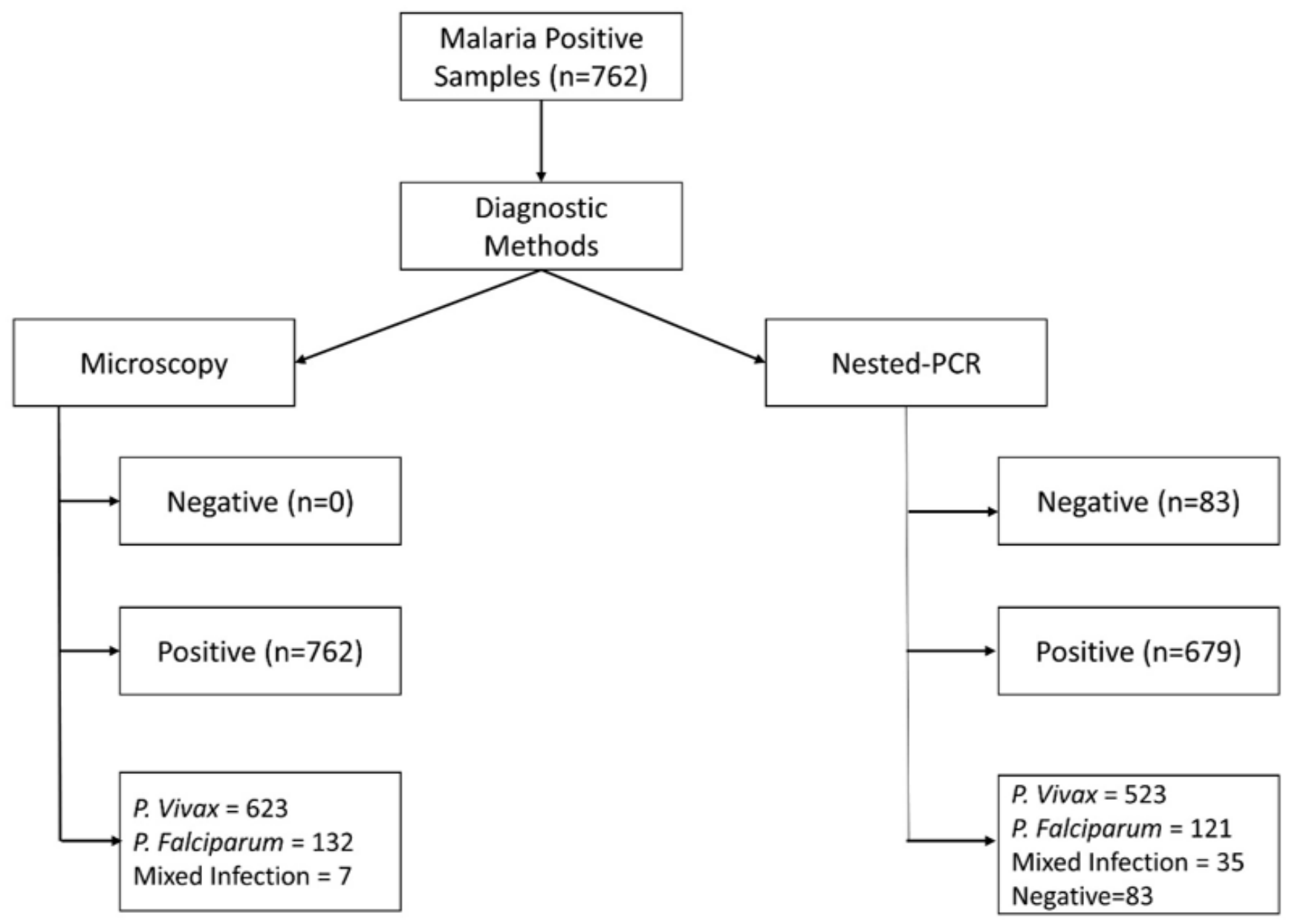

Figure 3 
Flow diagram of comparative analysis about diagnostic testing of $(n=762)$ malaria samples in two different methods. 